\title{
Dichloromethane as an Unusual Methylene Equivalent. A New Entry of Highly Nucleophilic and Selective Titanium-Methylene-Complexes for Ester Methylenation
}

Tu-Hsin Yan*, Ching-Ting Chien, Chia-Chung Tsai, Kuo-Wei Lin, and Yen-Hsien Wu

Department of Chemistry, National Chung-Hsing University, Taichung 400, Taiwan, Republic of China

E-mail: thyan@mail.nchu.edu.tw

\section{Supporting Information}

General Information. Dichloromethane was distilled from $\mathrm{P}_{2} \mathrm{O}_{5}$ prior to use. Commerically available esters were used as received (Lancaster, Acros, Aldrich, Tedia, RDH). Titanium tetrachloride was purchased from Fluka Chemie AG. Magnesium powder (ca 50 mesh) was purchased from Lancaster Synthesis Ltd. Esters $\mathbf{1 b},{ }^{1} \mathbf{1 c}-\mathbf{1 e},{ }^{2}$ and $\mathbf{1 g}-\mathbf{1} \mathbf{p}^{2}$ were prepared according to the reported procedudes. Flash chromatography was performed on on neutral (Mancherey Nagel) or basic aluminum oxide (Merck, 90-active basic). All reactions were carried out under an atmosphere of $\mathrm{N}_{2} .{ }^{1} \mathrm{H}$ and ${ }^{13} \mathrm{C} \mathrm{NMR}$ spectra were recorded on a Varian VXR-400 MHz spectrometer at ambient temperature. High-resolution mass spectra were determined on a Jeol JMS-HX 110 spectrometer.

${ }^{1}$ Stork, G.; Landesman, H. K. J. Am. Chem. Soc. 1956, 78, 5128.

${ }^{2}$ Neises, B.; Steglich, W. Angew. Chem., Int. Ed. Engl. 1978, 17, 522.

\section{General procedure for the methylenation of esters 1a-1e, 1g-1l, and 1p:}

A solution of ester $\mathbf{1}(1 \mathrm{mmol})$ in $2 \mathrm{~mL}$ of THF was added by syringe to a suspension consisting of $\mathrm{Mg}$ (192 mg, $8 \mathrm{mmol}), \mathrm{TiCl}_{4}(379 \mathrm{mg}, 0.21 \mathrm{~mL}, 2 \mathrm{mmol})$, and $\mathrm{CH}_{2} \mathrm{Cl}_{2}(5 \mathrm{~mL})$ at room temperature. After being stirred for $1 \mathrm{~h}$, the resulting black slurry was cooled to $0{ }^{\circ} \mathrm{C}$, then carefully poured into ice-cold saturated potassium carbonate solution $(10 \mathrm{~mL})$. The resulting mixture was stirred with $20 \mathrm{~mL}$ of ether and the phases were separated. After this procedure was repeated twice, the combined extracts were dried, evaporated, and purified by chromatography on basic aluminum oxide (elution with 100:3:1 hexane-ethyl acetate- $\mathrm{NEt}_{3}$ ).

Procedure for the methylenation of esters 1f: 
To a $0{ }^{\circ} \mathrm{C}$ suspension consisting of $\mathrm{Mg}$ (192 mg, $\left.8 \mathrm{mmol}\right), \mathrm{TiCl}_{4}(379 \mathrm{mg}, 0.21 \mathrm{~mL}, 2 \mathrm{mmol})$, and toluene $(5 \mathrm{~mL})$ was added dry THF $(4 \mathrm{~mL})$ over a 1-min period. After the initial exotherm had ceased, the cooling bath was removed. A solution of ester $\mathbf{1 f}(1 \mathrm{mmol})$ in $\mathrm{CH}_{2} \mathrm{Cl}_{2}(5 \mathrm{~mL})$ and THF $(1 \mathrm{~mL})$ was added. The reaction mixture was stirred for $1 \mathrm{~h}$ at room temperature. Standard workup and purification gave $\mathbf{2 f}$.

\section{General procedure for methylenation of esters $1 \mathrm{~m}-1 \mathrm{o}$ :}

Methylenation of benzyl 2,5-cyclohexadiene-1-carboxylate 1n: At $0{ }^{\circ} \mathrm{C}$, ester $\mathbf{1 n}(214 \mathrm{mg}$, $1 \mathrm{mmol})$ in $\mathrm{CH}_{2} \mathrm{Cl}_{2}(2 \mathrm{~mL})$ and THF $(2 \mathrm{~mL})$ was added to a suspension consisting of $\mathrm{Mg}$ (192 mg, $8 \mathrm{mmol}$ ) and $\mathrm{TiCl}_{4}\left(2 \mathrm{mmol}, 0.5 \mathrm{M}\right.$ in $\mathrm{CH}_{2} \mathrm{Cl}_{2}, 4 \mathrm{~mL}$ ). After the initial exotherm had ceased, the cooling bath was removed. The black slurry was stirred for $1 \mathrm{~h}$, then cooled to $0{ }^{\circ} \mathrm{C}$, and carefully poured into ice-cold saturated potassium carbonate solution $(10 \mathrm{~mL})$. The resulting mixture was stirred with $20 \mathrm{~mL}$ of ether and the phases were separated. After this procedure was repeated twice, the combined extracts were dried, evaporated, and purified by chromatography on basic aluminum oxide (elution with 100:3:1 hexane-ethyl acetate-NEt 3 ) to give vinyl ether $\mathbf{2 n}(191 \mathrm{mg}, 90 \%$ yield) as a colorless oil.

Methyl [2-(2methylenecyclohexyl)ethyl] vinyl ether 2b: As described above, $184 \mathrm{mg}$ (1 mmol) of $\mathbf{1 b}$ and $\mathrm{Mg} / \mathrm{TiCl}_{4} / \mathrm{CH}_{2} \mathrm{Cl}_{2} / \mathrm{THF}$ gave a crude reaction mixture. Purification by flash chromatography (silica gel, hexane) afforded $166 \mathrm{mg}(92 \%)$ of $\mathbf{2 b}$ as a colorless oil: ${ }^{1} \mathrm{H}$ NMR (400 MHz, $\left.\mathrm{C}_{6} \mathrm{D}_{6}\right) \delta 4.74$ and $4.71\left(2 \mathrm{~s}, 2 \mathrm{H}, H_{2} \mathrm{C}=\mathrm{C}\right), 3.97(\mathrm{~d}, J=1.2 \mathrm{~Hz}, 1 \mathrm{H}$, $H \mathrm{C}=\mathrm{C}-\mathrm{O}), 3.85(\mathrm{~d}, J=1.2 \mathrm{~Hz}, 1 \mathrm{H}, H \mathrm{C}=\mathrm{C}-\mathrm{O}), 3.23\left(\mathrm{~s}, 3 \mathrm{H}, H_{3} \mathrm{C}-\mathrm{O}\right), 5.63(\mathrm{dt}, J=10,2.8 \mathrm{~Hz}$, $1 \mathrm{H}, \mathrm{HC}=\mathrm{CH}), 4.41$ (bs, $1 \mathrm{H}, H \mathrm{C}-\mathrm{O}), 4.00$ and $4.02\left(2 \mathrm{~s}, 2 \mathrm{H}, H_{2} \mathrm{C}=\mathrm{C}-\mathrm{O}\right), 3.38(\mathrm{~s}, 2 \mathrm{H},=\mathrm{C}-$ $\left.H_{2} \mathrm{C}-\mathrm{Ph}\right), 2.24-1.92(\mathrm{~m}, 5 \mathrm{H}) 1.69-1.21(\mathrm{~m}, 8 \mathrm{H}) ;{ }^{13} \mathrm{C} \mathrm{NMR}\left(75.5 \mathrm{MHz}, \mathrm{C}_{6} \mathrm{D}_{6}\right) \delta 164.84$, $152.31,106.34,80.34,54.38,42.93,34.84,34.11,33.41,30.28,29.09$, 24.33; highresolution MS m/e Calcd for $\mathrm{C}_{12} \mathrm{H}_{20} \mathrm{O}:$ 180.1521. Found: 180.1514 .

(1-Benzylvinyl) phenyl ether 2e: As described above, $212 \mathrm{mg}(1 \mathrm{mmol})$ of $1 \mathrm{e}$ and $\mathrm{Mg} / \mathrm{TiCl}_{4} / \mathrm{CH}_{2} \mathrm{Cl}_{2} / \mathrm{THF}$ gave a crude reaction mixture. Purification by flash chromatography (silica gel, hexane) gave 2 e $(191 \mathrm{mg}, 90 \%)$ as a colorless oil: ${ }^{1} \mathrm{H}$ NMR (400 MHz, $\left.\mathrm{C}_{6} \mathrm{D}_{6}\right) \delta$ 7.26-6.83 (m, $\left.10 \mathrm{H}\right), 4.11$ (s, $\left.2 \mathrm{H}, \mathrm{H}_{2} \mathrm{C}=\mathrm{C}\right), 3.44\left(\mathrm{~s}, 2 \mathrm{H},=\mathrm{C}-\mathrm{H}_{2} \mathrm{C}-\mathrm{Ph}\right)$; ${ }^{13} \mathrm{C}$ NMR $\left(75.5 \mathrm{MHz}, \mathrm{C}_{6} \mathrm{D}_{6}\right) \delta 163.00,155.95,138.39,129.77,129.37,128.64,126.75$, 124.20, 121.22, 90.25, 40.97; high-resolution MS m/e Calcd for $\mathrm{C}_{15} \mathrm{H}_{14} \mathrm{O}$ : 210.1045 . Found: 210.1053.

3,4-Dihydro-2-methylene-2H-1-benzopyran 2f: As described above, $150 \mathrm{mg}$ (1 mmol) of 1f and $\mathrm{Mg} / \mathrm{TiCl}_{4} / \mathrm{CH}_{2} \mathrm{Cl}_{2} / \mathrm{THF}$ gave a crude reaction mixture. Purification by flash chromatography (silica gel, hexane) afforded $121 \mathrm{mg}(82 \%)$ of $\mathbf{2} \mathbf{f}$ as a colorless oil: ${ }^{1} \mathrm{H}$ NMR (400 MHz, $\left.\mathrm{C}_{6} \mathrm{D}_{6}\right) \delta$ 6.99-6.93 (m, $\left.2 \mathrm{H}\right), 6.80-6.74(\mathrm{~m}, 2 \mathrm{H}), 4.71$ and $3.99(2 \mathrm{~s}, 2 \mathrm{H}$, 
$\left.H_{2} \mathrm{C}=\mathrm{C}\right), 2.30\left(\mathrm{t}, J=6.4 \mathrm{~Hz}, 2 \mathrm{H}, \mathrm{H}_{2} \mathrm{C}-\mathrm{Ph}\right), 2.09$ (t, $\left.J=6.4 \mathrm{~Hz}, 2 \mathrm{H}, H_{2} \mathrm{C}-\mathrm{C}=\right) ; \quad{ }^{13} \mathrm{C}$ NMR $\left(75.5 \mathrm{MHz}, \mathrm{C}_{6} \mathrm{D}_{6}\right) \delta 156.46,153.63,128.94,125.80,122.99,121.41,116.47,89.60,26.53$, 25.33; high-resolution MS m/e Calcd for $\mathrm{C}_{9} \mathrm{H}_{8} \mathrm{O}_{2}$ : 148.0524. Found: 148.0531 .

(1-Benzylvinyl) 2-cyclohexenyl ether 2g: As described above, $216 \mathrm{mg}(1 \mathrm{mmol})$ of $\mathbf{1 g}$ and $\mathrm{Mg} / \mathrm{TiCl}_{4} / \mathrm{CH}_{2} \mathrm{Cl}_{2} / \mathrm{THF}$ gave a crude reaction mixture. Purification by flash chromatography (silica gel, hexane) gave $\mathbf{2 g}(161 \mathrm{mg}, 75 \%)$ as a colorless oil: ${ }^{1} \mathrm{H}$ NMR (400 MHz, $\left.\mathrm{C}_{6} \mathrm{D}_{6}\right) \delta$ 7.25-7.04 (m, $\left.5 \mathrm{H}, \mathrm{C}_{6} H_{5}\right), 5.85$ (dd, $\left.J=10,2.4 \mathrm{~Hz}, 1 \mathrm{H}, H \mathrm{C}=\mathrm{CH}\right), 5.63$ (dt, $J=10,2.8 \mathrm{~Hz}, 1 \mathrm{H}, \mathrm{HC}=\mathrm{CH}), 4.41$ (bs, $1 \mathrm{H}, \mathrm{HC}-\mathrm{O}), 4.00$ and $4.02\left(2 \mathrm{~s}, 2 \mathrm{H}, \mathrm{H}_{2} \mathrm{C}=\mathrm{C}-\mathrm{O}\right)$, $3.38\left(\mathrm{~s}, 2 \mathrm{H},=\mathrm{C}-\mathrm{H}_{2} \mathrm{C}-\mathrm{Ph}\right), 1.76-1.48(\mathrm{~m}, 5 \mathrm{H}), 1.32-1.22(\mathrm{~m}, 1 \mathrm{H}) ;{ }^{13} \mathrm{C}$ NMR $(75.5 \mathrm{MHz}$, $\left.\mathrm{C}_{6} \mathrm{D}_{6}\right) \delta 161.11,139.21,130.99,129.32,128.43,126.92,126.42,83.17,70.12,42.43,28.17$, 25.21, 19.41; high-resolution MS m/e Calcd for $\mathrm{C}_{15} \mathrm{H}_{18} \mathrm{O}$ : 214.1352. Found: 214.1358 .

2-Cyclohexenyl phenyl ether 2k: As described above, $212 \mathrm{mg}(1 \mathrm{mmol})$ of $\mathbf{1 k}$ and $\mathrm{Mg} / \mathrm{TiCl}_{4} / \mathrm{CH}_{2} \mathrm{Cl}_{2} / \mathrm{THF}$ gave a crude reaction mixture. Purification by flash chromatography (silica gel, hexane) afforded $164 \mathrm{mg}(78 \%)$ of $\mathbf{2 k}$ as a colorless oil: ${ }^{1} \mathrm{H}$ NMR (400 MHz, $\left.\mathrm{C}_{6} \mathrm{D}_{6}\right) \delta$ 7.76-7.74 (m, $\left.2 \mathrm{H}\right), 7.14-7.07$ (m, $\left.3 \mathrm{H}\right), 5.95$ (dd, $J=10,2.4 \mathrm{~Hz}, 1$ $\mathrm{H}, H \mathrm{C}=\mathrm{CH}), 5.71(\mathrm{dt}, J=10,2.8 \mathrm{~Hz}, 1 \mathrm{H}, \mathrm{HC}=\mathrm{CH}), 4.75(\mathrm{~d}, J=2.4 \mathrm{~Hz}, 1 \mathrm{H}, H \mathrm{C}=\mathrm{C}-\mathrm{O})$, 4.56 (bs, $1 \mathrm{H}, H \mathrm{C}-\mathrm{O}), 4.21(\mathrm{~d}, J=2.4 \mathrm{~Hz}, 1 \mathrm{H}, H \mathrm{C}=\mathrm{C}-\mathrm{O}), 1.89-1.60(\mathrm{~m}, 5 \mathrm{H}), 1.36-1.30(\mathrm{~m}$, $1 \mathrm{H}) ;{ }^{13} \mathrm{C}$ NMR $\left(75.5 \mathrm{MHz}, \mathrm{C}_{6} \mathrm{D}_{6}\right) \delta 158.89,137.74,131.31,128.54,128.29,126.81$, 126.03, 83.27, 70.46, 28.31, 25.31, 19.46; high-resolution MS m/e Calcd for $\mathrm{C}_{15} \mathrm{H}_{14} \mathrm{O}$ : 210.1045. Found: 210.1053.

Methyl 1-(2,5-cyclohexadienyl)vinyl ether 2m: As described above, $138 \mathrm{mg}$ (1 mmol) of $1 \mathbf{m}$ and $\mathrm{Mg} / \mathrm{TiCl}_{4} / \mathrm{CH}_{2} \mathrm{Cl}_{2} / \mathrm{THF}$ gave a crude reaction mixture. Purification by flash chromatography (silica gel, hexane) gave $\mathbf{2 m}(119 \mathrm{mg}, 88 \%)$ as a colorless oil: ${ }^{1} \mathrm{H}$ NMR $\left(400 \mathrm{MHz}, \mathrm{C}_{6} \mathrm{D}_{6}\right) \delta$ 5.89-5.86 (m, $\left.2 \mathrm{H},(H \mathrm{C}=\mathrm{C})_{2} \mathrm{CH}\right), 5.66-5.63\left(\mathrm{~m}, 2 \mathrm{H},(H \mathrm{C}=\mathrm{C})_{2} \mathrm{CH}_{2}\right), 4.06$ $(\mathrm{d}, J=1.6 \mathrm{~Hz}, 1 \mathrm{H}, H \mathrm{C}=\mathrm{C}-\mathrm{O}), 3.88(\mathrm{~d}, J=1.6 \mathrm{~Hz}, 1 \mathrm{H}, H \mathrm{C}=\mathrm{C}-\mathrm{O}), 3.58-3.55(\mathrm{~m}, 1 \mathrm{H}, H \mathrm{C}-$ $\mathrm{C}=\mathrm{C}), 3.19$ (s, $\left.3 \mathrm{H}, \mathrm{H}_{3} \mathrm{C}-\mathrm{O}\right), 2.48-2.45\left(\mathrm{~m}, 2 \mathrm{H}, \mathrm{H}_{2} \mathrm{C}-\mathrm{C}=\mathrm{C}\right) ; \quad{ }^{13} \mathrm{C} \mathrm{NMR}\left(75.5 \mathrm{MHz}, \mathrm{C}_{6} \mathrm{D}_{6}\right) \delta$ 166.64, 126.62, 124.78, 80.72, 54.60, 41.57, 26.17; high-resolution MS m/e Calcd for $\mathrm{C}_{9} \mathrm{H}_{12} \mathrm{O}:$ 136.0880. Found: 136.0884 .

Benzyl 1-(2,5-cyclohexadienyl)vinyl ether 2n: As described above, $214 \mathrm{mg}$ (1 mmol) of 1n and $\mathrm{Mg} / \mathrm{TiCl}_{4} / \mathrm{CH}_{2} \mathrm{Cl}_{2} / \mathrm{THF}$ gave a crude reaction mixture. Purification by flash chromatography (silica gel, hexane) afforded $190 \mathrm{mg}(90 \%)$ of $\mathbf{2 n}$ as a colorless oil: ${ }^{1} \mathrm{H}$ NMR (400 MHz, $\left.\mathrm{C}_{6} \mathrm{D}_{6}\right) \delta$ 7.22-7.04 (m, $\left.5 \mathrm{H}, \mathrm{C}_{6} H_{5}\right)$, 5.94-5.90 (m, $\left.2 \mathrm{H},(H \mathrm{C}=\mathrm{C}){ }_{2} \mathrm{CH}\right), 5.67-$ $5.63\left(\mathrm{~m}, 2 \mathrm{H},(H \mathrm{C}=\mathrm{C})_{2} \mathrm{CH}_{2}\right), 4.53\left(\mathrm{~s}, 2 \mathrm{H}, H_{2} \mathrm{C}-\mathrm{O}\right), 4.15(\mathrm{~d}, J=1.6 \mathrm{~Hz}, 1 \mathrm{H}, H \mathrm{C}=\mathrm{C}-\mathrm{O}), 4.00$ $(\mathrm{d}, J=1.6 \mathrm{~Hz}, 1 \mathrm{H}, H \mathrm{C}=\mathrm{C}-\mathrm{O}), 3.63-3.60(\mathrm{~m}, 1 \mathrm{H}, H \mathrm{C}-\mathrm{C}=\mathrm{C}), 2.46-2.00\left(\mathrm{~m}, 2 \mathrm{H}, H_{2} \mathrm{C}-\mathrm{C}=\mathrm{C}\right)$; ${ }^{13} \mathrm{C}$ NMR $\left(75.5 \mathrm{MHz}, \mathrm{C}_{6} \mathrm{D}_{6}\right) \delta 165.43,137.73,128.69,127.76,127.56,126.52,124.90$, 
82.25, 69.59, 41.55, 26.21; high-resolution MS m/e Calcd for $\mathrm{C}_{15} \mathrm{H}_{16} \mathrm{O}$ : 212.1209 . Found: 212.1205. Anal. Calcd for $\mathrm{C}_{15} \mathrm{H}_{16} \mathrm{O}$ : C, 84.87; H, 7.60. Found: C, 84.63; H, 7.66.

Isopropyl 1-(2,5-cyclohexadienyl)vinyl ether 2o: As described above, $166 \mathrm{mg}$ (1 mmol) of 10 and $\mathrm{Mg} / \mathrm{TiCl}_{4} / \mathrm{CH}_{2} \mathrm{Cl}_{2} / \mathrm{THF}$ gave a crude reaction mixture. Purification by flash chromatography ( silica gel, hexane) gave $20(142 \mathrm{mg}, 87 \%)$ as a colorless oil: ${ }^{1} \mathrm{H}$ NMR $\left(400 \mathrm{MHz}, \mathrm{C}_{6} \mathrm{D}_{6}\right)$ 85.92-5.89 (m, $\left.2 \mathrm{H},(H \mathrm{C}=\mathrm{C})_{2} \mathrm{CH}\right), 5.67-5.63\left(\mathrm{~m}, 2 \mathrm{H},(H \mathrm{C}=\mathrm{C})_{2} \mathrm{CH}_{2}\right), 4.15$ $(\mathrm{d}, J=2.0 \mathrm{~Hz}, 1 \mathrm{H}, H \mathrm{C}=\mathrm{C}-\mathrm{O}), 4.04$ (septet, $\left.J=6.0 \mathrm{~Hz}, 1 \mathrm{H}, H \mathrm{C}\left(\mathrm{CH}_{3}\right)_{2}\right), 3.92(\mathrm{~d}, J=2.0 \mathrm{~Hz}$, $1 \mathrm{H}, H \mathrm{C}=\mathrm{C}-\mathrm{O}), 3.51-3.56(\mathrm{~m}, 1 \mathrm{H}, H \mathrm{C}-\mathrm{C}=\mathrm{C}), 2.53-2.43\left(\mathrm{~m}, 2 \mathrm{H}, H_{2} \mathrm{C}-\mathrm{C}=\mathrm{C}\right), 1.07(\mathrm{~d}, J=6.0$ $\left.\mathrm{Hz}, 6 \mathrm{H},\left(\mathrm{CH}_{3}\right)_{2} \mathrm{C}\right) ;{ }^{13} \mathrm{C} \mathrm{NMR}\left(75.5 \mathrm{MHz}, \mathrm{C}_{6} \mathrm{D}_{6}\right) \delta 164.07,126.84,124.59,81.58,68.55$, 41.83, 26.27, 21.53; high-resolution MS m/e Calcd for $\mathrm{C}_{11} \mathrm{H}_{16} \mathrm{O}:$ 164.1215. Found: 164.1208 .

2-t-Butoxy-4-phenylbutene 2p: As described above, $206 \mathrm{mg}(1 \mathrm{mmol})$ of $\mathbf{1 p}$ and $\mathrm{Mg} / \mathrm{TiCl}_{4} / \mathrm{CH}_{2} \mathrm{Cl}_{2} / \mathrm{THF}$ gave a crude reaction mixture. Purification by flash chromatography (silica gel, hexane) afforded $103 \mathrm{mg}(50 \%)$ of $\mathbf{2 p}$ as a colorless oil: ${ }^{1} \mathrm{H}$ NMR (400 MHz, $\left.\mathrm{C}_{6} \mathrm{D}_{6}\right) \delta$ 7.15-7.04 (m, $\left.5 \mathrm{H}\right), 4.03$ and $4.00\left(2 \mathrm{~s}, 2 \mathrm{H}, \mathrm{H}_{2} \mathrm{C}=\mathrm{C}\right), 2.81$ (t, $J=$ $\left.8.0 \mathrm{~Hz}, 2 \mathrm{H}, \mathrm{H}_{2} \mathrm{C}-\mathrm{Ph}\right), 2.35\left(\mathrm{t}, J=8.0 \mathrm{~Hz}, 2 \mathrm{H}, \mathrm{H}_{2} \mathrm{C}-\mathrm{C}=\right), 1.26\left(\mathrm{~s}, 9 \mathrm{H},\left(\mathrm{H}_{3} \mathrm{C}\right)_{3}-\mathrm{C}\right) ;{ }^{13} \mathrm{C}$ NMR (75.5 MHz, $\left.\mathrm{C}_{6} \mathrm{D}_{6}\right) \delta 158.79,142.22,128.78,128.58,126.00,87.01,76.45,39.54$, 34.45, 28.28; high-resolution $\mathrm{MS} \mathrm{m} / \mathrm{e}$ Calcd for $\mathrm{C}_{14} \mathrm{H}_{20} \mathrm{O}$ : 204.1500. Found: 204.1507. 